\title{
Variability of Metabolic Power Data in Elite Soccer Players During Pre-Season Matches
}

\author{
by \\ Matthias Wilhelm Hoppe ${ }^{1}$, Christian Baumgart ${ }^{1}$, Mirko Slomka², \\ Ted Polglaze ${ }^{3}$, Jürgen Freiwald ${ }^{1}$
}

This study aimed to determine the within-subject variability of GPS-derived metabolic power data in elite soccer players across several pre-season matches and compare the variability of high metabolic power, velocity, acceleration and deceleration running. Additionally, differences in metabolic power data among playing positions and relationships with various physical abilities were also investigated. Metabolic power data from 12 outfield starting players competing in the German Bundesliga were collected during five pre-season matches using GPS-technology (10 $\mathrm{Hz}$ ). The players were also tested for speed, agility, power and intermittent endurance. Variability of global metabolic power data such as energy expenditure $(\mathrm{CV}=2.2-7.0 \%)$ was lower than that for high-intensity including time $\geq 20$ $\mathrm{W} \cdot \mathrm{kg}^{-1}(\mathrm{CV}=14.0-26.2 \%)$. Variability of high metabolic power $\left(\geq 20 \mathrm{~W} \cdot \mathrm{kg}^{-1} ; \mathrm{CV}=14.1 \pm 3.5 \%\right)$ was comparable to that of high velocity $\left(\geq 15.5 \mathrm{~km} \cdot \mathrm{h}^{-1} ; \mathrm{CV}=17.0 \pm 6.2 \%\right)$, acceleration $\left(\geq 3 \mathrm{~m} \cdot \mathrm{s}^{-2} ; \mathrm{CV}=11.1 \pm 5.1 \%\right)$ and deceleration running $\left(\leq-3 \mathrm{~m} \cdot \mathrm{s}^{-2} ; C V=11.9 \pm 4.5 \%\right)(p>0.05, E S<0.2)$. Defenders had a largely higher overall energy expenditure than midfielders and attackers $(p<0.01, E S>0.6)$. Overall energy expenditure and cost were largely to very largely correlated with $5 \mathrm{~m}$ speed and $22 \mathrm{~m}$ agility sprint time and counter movement jump height $(r=-0.70-0.69, p<0.05)$. The detected variability indicates that global GPS-derived metabolic power data in elite soccer players from a single preseason match should be preferably used for practical applications. Contrary, high-intensity indicators should be interpreted cautiously and repeated match observations are recommended to establish meaningful high-intensity profiles of the players. Differences among playing positions and relationships with explosive physical abilities indicate that metabolic power analyses can provide new insights into the mechanics and energetics of soccer.

Key words: data filter; global positioning system; interval shuttle run test; time motion analysis.

\section{Introduction}

During the past few decades, the extensive application of time-motion analyses has substantially increased the knowledge of match running demands for elite soccer players (Hoppe et al., 2015b). In most studies, video or global position system (GPS) derived time-motion data were collected to determine the time spent, distance covered or frequency in various velocity categories (Carling et al., 2008). However, for soccer, this velocity based approach is potentially not appropriate due to the frequent accelerations and decelerations performed over short distances, where the velocities attained are not high (Dwyer and Gabbett, 2012). Consequently, when the velocity as a criterion is used, the mechanical and metabolic loads of soccer players experienced during play may be underestimated (Polglaze et al., 2016).

To address these limitations, di Prampero et al. (2005) suggested a novel approach to

1 - University of Wuppertal, Department of Movement and Training Science, Wuppertal, Germany.

2 - Former Head Coach of FC Schalke 04, Hannover 96, and Hamburger SV, Hannover, Germany.

3 - University of Western Australia, School of Sport Science, Exercise and Health, Perth, Australia. 
analyze time-motion data grounded on the assumption that accelerated/decelerated running on a horizontal level is energetically equivalent to uphill/downhill running at a constant velocity on an 'equivalent slope'. Since the energetics of uphill/downhill running are known and energy costs are independent of the velocity, the energy costs of accelerated/decelerated running on a horizontal level can be estimated. This energy cost can then be multiplied with the velocity to obtain the metabolic power ( $\mathrm{P}_{\mathrm{met}}$ ) (di Prampero et al., 2015). Thus, when the velocity, acceleration and deceleration from time-motion data are derived, the instantaneous $\mathrm{P}_{\mathrm{met}}$ can be computed, providing a promising basis to more comprehensively examine the match running demands of soccer players (Manzi et al., 2014; Osgnach et al., 2010).

Presently, only two studies exist, which have applied this novel energetic approach to soccer matches. Osgnach et al. (2010) were the first to investigate the running demands of Italian Serie A players through $P_{\text {met }}$ analyses. The results show that $18 \%$ of the total distance covered was considered high-intensity when using velocity criteria, but that figure was $26 \%$ when using equivalent $P_{m e t}$ thresholds. Recently, Manzi et al. (2014) examined the relationships between Pmet data and key variables of aerobic fitness in Italian Serie A players and found a moderate correlation between maximum oxygen uptake and distance covered at high Pmet. Overall, these studies show that a velocity approach indeed underestimates the "true" match running demands of soccer and support the ecological validity of aerobic fitness in elite players. However, more research investigating Pmet data is needed (Polglaze et al., 2016).

In elite soccer players, the match-to-match variability of high velocity running is large (Gregson et al., 2010; Rampinini et al., 2007). Furthermore, the reliability of GPS-technology for measuring high acceleration and deceleration running has been questioned by two studies (Buchheit et al., 2014a, 2014b). Consequently, and due to the basis of the $P_{\text {met }}$ calculations, it is necessary to determine the within-subject variability of GPS-derived Pmet data across several matches and compare the variability of high $P_{\text {met, }}$ velocity, acceleration and deceleration running, which is important to judge, for example, above which threshold effects are meaningful (Hopkins, 2000; Hopkins et al., 2009). Additionally, it is useful to identify differences among playing positions not only in velocity data, being well described in the literature (Bradley et al., 2009; Di Salvo et al., 2007), but also in Pmet data. Moreover, it is of significance to investigate the relationships between $P_{\text {met }}$ data and important physical abilities for soccer players beside aerobic fitness (Manzi et al., 2014), such as speed, agility, power and intermittent endurance (Boone et al., 2012; Haugen et al., 2013; Hoppe et al., 2013). Such knowledge will provide new insights into the mechanics and energetics of soccer, which may help to optimize training drills, testing procedures and nutritional recommendations for the players.

The purpose of this study was to determine the within-subject variability of GPSderived $P_{\text {met }}$ data in elite soccer players across several pre-season matches and compare the variability of high $P_{\text {met, }}$ velocity, acceleration and deceleration running. Additionally, differences in $P_{\text {met }}$ data among playing positions and relationships with various physical abilities were also investigated.

\section{Methods}

\section{Participants}

Twelve soccer players competing in the German Bundesliga and UEFA Europa League participated in this study. All players were from the same team, which ranked in the final topseven of the Bundesliga and reached the quarterfinals of the Europa League. Eight of the players were also playing for their national teams. The sample included only outfield players, who were primarily starters. Since nonstarters and talented youth players from the $2^{\text {nd }}$ team were excluded, a homogeneous sample of "true" elite players was investigated. The players were assigned to one of the three following groups according to their playing position: defenders $(n=$ $4)$, midfielders $(n=5)$ and attackers $(n=3)$. Table 2 shows the anthropometric characteristics of the players. The study was approved by the Ethics Committee of the University of Wuppertal.

\section{Experimental design}

All testing procedures were conducted at the end of the pre-season, where all players had attained a stable fitness level. On one day, the players were tested for anthropometric 
characteristics (body height, mass, fat and fat-free mass), speed and agility (sprint times), power (counter movement jump height) and intermittent endurance (interval shuttle run test distance). Over the subsequent 15 days, $P_{m e t}$ data from five pre-season matches against opponents all competing in highest leagues were derived from GPS-based time-motion analyses. Since the players did not play all matches over the entire 90 min due to numerous substitutions performed by the coaches to fine-tune the team tactics, only the data of fully completed halves were considered (mean playing time: $45 \pm 1 \mathrm{~min}$ ), resulting in a total of 61 halves analyzed (range: 4-6 halves per player).

\section{Anthropometric characteristics}

Body fat and fat-free mass of the players were assessed using a 4-point bioelectric impedance analysis in a supine position (Bodystat, QuadScan 4000, Douglas, United Kingdom) (Hoppe et al., 2013). Thereafter, the players performed a warm-up, which was conducted by conditioning coaches.

\section{Physical abilities}

All speed, agility, power and intermittent endurance tests were performed as previously described (Hoppe et al., 2013, 2015a). Briefly, for speed, the players started from a contact plate and sprint times were recorded with timing gates (TDS Werthner Sport Consulting, Linz, Austria) at 5, 10, 20 and $30 \mathrm{~m}$. For agility, the players started $1 \mathrm{~m}$ before the first timing gate (hs-electronics, Falkensee, Germany) and sprinted over $22 \mathrm{~m}$ including four changes of direction (Figure 1). For power, the players performed a counter movement jump with an arm swing during which the height was determined using two force plates (Kistler, 9286AA, Winterthur, Switzerland). Each test was repeated three times and the means were used for statistical analyses.

Finally, for intermittent endurance, the players performed an interval shuttle run test (Lemmink et al., 2004), whereby they ran $20 \mathrm{~m}$ shuttles at progressively increasing velocities controlled by audio signals. The test was terminated, when the players could not follow the pace during two consecutive shuttles. To clarify whether maximum exertion was reached, the players were equipped with heart rate monitors (Suunto, t6, Vantaa, Finland) and required to reach $>95 \%$ of their individual maximum heart rate, which was met in all cases. The number of shuttles completed was used for statistical analyses.

\section{Metabolic power data}

The $P_{\text {met }}$ data were assessed employing portable GPS-devices, sampling positional data at $10 \mathrm{~Hz}$ (Catapult Innovations, MinimaxX S4, Melbourne, Australia). For these devices, acceptable accuracy and reliability for measuring velocity (Castellano et al., 2011), acceleration and deceleration (Varley et al., 2012) and Pmet data (Rampinini et al., 2015) had been provided. The devices were worn in neoprene harnesses located between both scapulae. To avoid inter-unit errors, the players wore the same device for each match (Akenhead et al., 2014). During the matches, the devices had connections with $11.8 \pm 0.5$ satellites and the horizontal dilution of the position was 1.1 \pm 0.1, indicating good measuring conditions (Varley et al., 2012). The proprietary software (Catapult Innovations, Sprint 5.1.4., Melbourne, Australia) provided the raw velocity data, which were first passed through a Butterworth filter (1 $\mathrm{Hz}$ with 2 passes) to eliminate noise (Figure 2) (Hoppe et al., 2014). Then, the velocity data were exported to a spreadsheet (Microsoft, Excel 2013, Redmond, USA), which calculated the associated acceleration and deceleration data. Grounded thereon, the $\mathrm{P}_{\text {met }}$ data were computed according to Osgnach et al. (2010). Specifically, the following global and high-intensity measures were considered: total distance covered, energy expenditure per unit body mass and overall, energy cost (i.e., energy expenditure per unit body mass and distance covered), mean $\mathrm{P}_{\text {met, }}$ time spent at five $P_{\text {met }}$ categories ( 0 to $<10,10$ to $<20,20$ to $<35,35$ to $<55$ and $\left.\geq 55 \mathrm{~W} \cdot \mathrm{kg}^{-1}\right)$ and peak $P_{\text {met. }}$. The time spent $\geq 20 \mathrm{~W} \cdot \mathrm{kg}^{-1}$ was defined as high $P_{\text {met }}$ running, as this value is energetically equivalent to an oxygen uptake of $57 \mathrm{ml} \cdot \mathrm{kg}^{-1} \cdot \mathrm{min}^{-1}$ (above resting) (Osgnach et al., 2010), approximately corresponding to the average maximum oxygen uptake of players with a body mass of $78 \mathrm{~kg}$ (Hoppe et al., 2013). The time spent $\geq 15.5 \mathrm{~km} \cdot \mathrm{h}^{-1}$ was defined as high velocity running, since continuous running at this velocity is energetically comparable to accelerated/decelerated running at $20 \mathrm{~W} \cdot \mathrm{kg}^{-1}$ (Osgnach et al., 2010). Lastly, the time spent $\geq+3$ and $\leq-3 \mathrm{~m} \cdot \mathrm{s}^{-2}$ was defined as high acceleration and deceleration running, respectively, as previously 
described (Osgnach et al., 2010).

\section{Statistical analyses}

The Kolmogorov-Smirnov test revealed that all data were normally distributed. However, due to the small sample size, non-parametric follow-up tests were consistently applied (Pallant, 2010). To determine the within-subject variability of $P_{\text {met }}$ data, the means and standard deviations of the players across the matches were calculated and expressed as the overall coefficient of variation (CV) (Hopkins, 2000). To compare the variability of high $P_{\text {met, }}$ velocity, acceleration and deceleration running, the time spent above the associated thresholds was expressed as CVs likewise. The differences in CVs and also in the time spent above the thresholds were investigated using Friedman and Wilcoxon-Signed-Rank tests for post-hoc analyses. To identify differences in $P_{\text {met }}$ data among the playing positions, the average values of the players across the matches were analyzed with a Kruskal-Wallis and MannWhitney-U test for post-hoc analyses. The latter two statistical tests were also applied to detect differences in anthropometric characteristics and physical abilities among the playing positions. To interpret the meaningfulness of the differences in $\mathrm{CVs}$ and further mean values, non-parametric follow-up test associated effect sizes (ES) were calculated through dividing the $\mathrm{z}$-values by the square root of the number of cases (Pallant, 2010) and were interpreted using the following qualitative descriptors: 0.1 to $<0.3$, small; 0.3 to $<0.5$, moderate and $\geq 0.5$, large (Pallant, 2010). Finally, to investigate the relationships between $P_{m e t}$ data and physical abilities, Spearman rank correlation coefficients $(r)$ were computed and interpreted accordingly: $<0.1$, trivial; 0.1 to $<0.3$, small; 0.3 to $<0.5$, moderate; 0.5 to $<0.7$, large; 0.7 to $<0.9$, very large and $\geq 0.9$, extremely large (Hopkins et al., 2009). The threshold for global significance was set at $p<0.05$, whereas Bonferroni adjustments were applied to control for type 1 errors during post-hoc testing (Pallant, 2010). The SPSS software (IBM, Version 22, New York, USA) was used for all statistical calculations.

\section{Results}

\section{Within-subject variability}

Table 1 summarizes the CVs for all $\mathrm{P}_{\text {met }}$ data. The CVs ranged from 2.2-26.2\%.

Figure $3 \mathrm{~A}$ shows the $\mathrm{CVs}$ for high $\mathrm{P}_{\mathrm{met}}$ velocity, acceleration and deceleration running. The corresponding values were $14.1 \pm 3.5 \%, 17.0 \pm$ $6.2 \%, 11.1 \pm 5.1 \%$ and $11.9 \pm 4.5 \%$, respectively. The CV for high Pmet running was comparable to that for high velocity, acceleration and deceleration running $(p>0.05, E S<0.2)$. However, the $\mathrm{CV}$ for high velocity was moderately higher than that for high acceleration and deceleration running $(p<0.01, E S=0.3$ ). Figure 3B displays the time spent at high $P_{\text {met, }}$ velocity, acceleration and deceleration running. The players spent moderately more time at high Pmet than at high velocity, acceleration and deceleration running $(p$ $<0.01$, ES $=0.4$ ) and moderately more time was spent at high velocity than at high acceleration and deceleration running $(p<0.01, E S=0.4)$. The time spent at high acceleration and deceleration running did not differ $(p=0.36, E S=0.1)$.

\section{Differences among playing positions}

Table 2 provides the differences in $P_{\text {met }}$ data, anthropometric characteristics and physical abilities among playing positions. Defenders had a largely higher overall energy expenditure than midfielders and attackers $(p<0.01, E S>0.6)$. Defenders also had a largely greater body height, mass and fat-free mass $(p<0.01, E S>0.6)$. No further differences were evident $(p>0.06)$.

\section{Relationships}

Figure 4 illustrates the relationships found between $P_{\text {met }}$ data and physical abilities. Overall energy expenditure was largely correlated with 5 $\mathrm{m}$ speed sprint time $(r=0.63, p=0.03$; Figure $4 \mathrm{~A})$ and counter movement jump height $(r=-0.66, p=$ 0.02; Figure 4B). Energy cost was largely correlated with $22 \mathrm{~m}$ agility sprint time $(r=0.69, p$ $=0.01$; Figure $4 \mathrm{C}$ ) and very largely with counter movement jump height $(r=-0.70, p=0.01$; Figure $4 \mathrm{D})$. No further relationships were detected $(p>$ 0.09).

\section{Discussion}

The main findings were: (1) variability of global GPS-derived $P_{\text {met }}$ data was lower than that for high-intensity; (2) variability of high $P_{\text {met }}$ was comparable to that of high velocity, acceleration and deceleration running; (3) defenders had a largely higher overall energy expenditure than midfielders and attackers and (4) overall energy expenditure and cost were largely to very largely correlated with explosive physical abilities. 


\section{Table 1}

Within-subject variability of metabolic power data in elite soccer players $(n=$ 12) during five pre-season matches. Variability of the total distance covered is also shown.

\begin{tabular}{lcc}
\hline \multicolumn{1}{c}{ Variable } & \multicolumn{2}{c}{ Coefficient of variation $(\%)$} \\
\hline Total distance covered $(\mathrm{m})$ & $5.8 \pm 2.0$ & $2.3-9.1$ \\
Overall energy expenditure $(\mathrm{kJ})$ & $5.6 \pm 1.8$ & $2.9-8.5$ \\
Energy expenditure $\left(\mathrm{kJ} \cdot \mathrm{kg}^{-1}\right)$ & $5.9 \pm 1.8$ & $2.9-8.5$ \\
Energy cost $\left(\mathrm{J} \cdot \mathrm{kg}^{-1} \cdot \mathrm{m}^{-1}\right)$ & $2.2 \pm 2.9$ & $0.8-11.4$ \\
Mean metabolic power $\left(\mathrm{W} \cdot \mathrm{kg}^{-1}\right)$ & $7.0 \pm 1.4$ & $5.0-9.5$ \\
0 to $<10 \mathrm{~W} \cdot \mathrm{kg}^{-1}(\mathrm{~s})$ & $5.9 \pm 1.5$ & $4.2-8.4$ \\
10 to $<20 \mathrm{~W} \cdot \mathrm{kg}^{-1}(\mathrm{~s})$ & $11.2 \pm 4.1$ & $5.1-17.6$ \\
20 to $<35 \mathrm{~W} \cdot \mathrm{kg}^{-1}(\mathrm{~s})$ & $14.0 \pm 4.4$ & $6.1-21.8$ \\
35 to $<55 \mathrm{~W} \cdot \mathrm{kg}^{-1}(\mathrm{~s})$ & $14.2 \pm 2.9$ & $11.4-22.0$ \\
$\geq 55 \mathrm{~W} \cdot \mathrm{kg}^{-1}(\mathrm{~s})$ & $19.3 \pm 8.3$ & $10.6-31.8$ \\
Peak metabolic power $\left(\mathrm{W} \cdot \mathrm{kg}^{-1}\right)$ & $26.2 \pm 13.0$ & $11.9-50.0$ \\
\hline
\end{tabular}

Values presented are mean \pm standard deviation and range.

\section{Table 2}

Metabolic power data during five pre-season matches, anthropometric characteristics and physical abilities of elite soccer players $(n=12)$ according to their playing positions. Total distance covered and time spent at high velocity, acceleration and deceleration running are also shown.

\begin{tabular}{|c|c|c|c|c|}
\hline Variable & $\begin{array}{c}\text { All } \\
(n=12)\end{array}$ & $\begin{array}{c}\text { Defenders } \\
(n=4)\end{array}$ & $\begin{array}{c}\text { Midfielders } \\
(n=5)\end{array}$ & $\begin{array}{c}\text { Attackers } \\
(n=3)\end{array}$ \\
\hline Total distance covered $(\mathrm{m})$ & $5137 \pm 356$ & $4997 \pm 381$ & $5221 \pm 354$ & $5118 \pm 411$ \\
\hline Overall energy expenditure (kJ) & $1936 \pm 162$ & $2103 \pm 69^{*}$ & $1854 \pm 116$ & $1849 \pm 160$ \\
\hline Energy expenditure $\left(\mathrm{kJ} \cdot \mathrm{kg}^{-1}\right)$ & $24.2 \pm 1.4$ & $24.0 \pm 1.1$ & $24.3 \pm 1.6$ & $24.1 \pm 2.0$ \\
\hline Energy cost $\left(\mathrm{J} \cdot \mathrm{kg}^{-1} \cdot \mathrm{m}^{-1}\right)$ & $4.7 \pm 0.1$ & $4.8 \pm 0.2$ & $4.7 \pm 0.1$ & $4.7 \pm 0.0$ \\
\hline Mean metabolic power $\left(\mathrm{W} \cdot \mathrm{kg}^{-1}\right)$ & $8.5 \pm 0.6$ & $8.3 \pm 0.6$ & $8.6 \pm 0.6$ & $8.6 \pm 0.7$ \\
\hline 0 to $<10 \mathrm{~W} \cdot \mathrm{kg}^{-1}(\mathrm{~s})$ & $2057 \pm 89$ & $2063 \pm 56$ & $2056 \pm 111$ & $2050 \pm 116$ \\
\hline 10 to $<20 \mathrm{~W} \cdot \mathrm{kg}^{-1}(\mathrm{~s})$ & $542 \pm 56$ & $541 \pm 50$ & $548 \pm 69$ & $536 \pm 63$ \\
\hline 20 to $<35 \mathrm{~W} \cdot \mathrm{kg}^{-1}$ (s) & $154 \pm 27$ & $149 \pm 26$ & $157 \pm 28$ & $155 \pm 35$ \\
\hline 35 to $<55 \mathrm{~W} \cdot \mathrm{kg}^{-1}(\mathrm{~s})$ & $40 \pm 7$ & $37 \pm 9$ & $41 \pm 6$ & $41 \pm 9$ \\
\hline$\geq 55 \mathrm{~W} \cdot \mathrm{kg}^{-1}(\mathrm{~s})$ & $12 \pm 2$ & $11 \pm 2$ & $13 \pm 2$ & $14 \pm 2$ \\
\hline Peak metabolic power $\left(\mathrm{W} \cdot \mathrm{kg}^{-1}\right)$ & $161.6 \pm 29.9$ & $145.7 \pm 20.1$ & $157.7 \pm 26.7$ & $189.4 \pm 34.3$ \\
\hline High metabolic power $\left(\geq 20 \mathrm{~W} \cdot \mathrm{kg}^{-1}\right)(\mathrm{s})$ & $206 \pm 34$ & $196 \pm 35$ & $212 \pm 33$ & $209 \pm 45$ \\
\hline High velocity $\left(\geq 15.5 \mathrm{~km} \cdot \mathrm{h}^{-1}\right)(\mathrm{s})$ & $128 \pm 27$ & $114 \pm 29$ & $135 \pm 22$ & $138 \pm 37$ \\
\hline High acceleration $\left(\geq 3 \mathrm{~m} \cdot \mathrm{s}^{-2}\right)(\mathrm{s})$ & $16 \pm 2$ & $17 \pm 2$ & $15 \pm 1$ & $14 \pm 1$ \\
\hline High deceleration $\left(\leq-3 \mathrm{~m} \cdot \mathrm{s}^{-2}\right)(\mathrm{s})$ & $15 \pm 2$ & $16 \pm 1$ & $15 \pm 2$ & $15 \pm 2$ \\
\hline Age (years) & $26 \pm 3$ & $28 \pm 1$ & $24 \pm 3$ & $25 \pm 2$ \\
\hline Body height (m) & $1.82 \pm 0.06$ & $1.89 \pm 0.02^{*}$ & $1.78 \pm 0.04$ & $1.80 \pm 0.02$ \\
\hline Body mass (kg) & $80.2 \pm 6.2$ & $87.7 \pm 3.3^{*}$ & $76.4 \pm 2.8$ & $76.6 \pm 3.3$ \\
\hline Body fat (\%) & $10.5 \pm 2.2$ & $11.1 \pm 3.5$ & $9.9 \pm 1.9$ & $10.7 \pm 0.1$ \\
\hline Body fat-free mass $(\mathrm{kg})$ & $71.3 \pm 5.7$ & $77.9 \pm 3.4^{*}$ & $68.1 \pm 3.7$ & $67.7 \pm 2.0$ \\
\hline $5 \mathrm{~m}$ speed sprint time (s) & $1.07 \pm 0.03$ & $1.10 \pm 0.03$ & $1.06 \pm 0.02$ & $1.06 \pm 0.02$ \\
\hline $10 \mathrm{~m}$ speed sprint time $(\mathrm{s})$ & $1.80 \pm 0.04$ & $1.84 \pm 0.04$ & $1.79 \pm 0.03$ & $1.79 \pm 0.05$ \\
\hline $20 \mathrm{~m}$ speed sprint time $(\mathrm{s})$ & $3.06 \pm 0.07$ & $3.11 \pm 0.06$ & $3.04 \pm 0.06$ & $3.04 \pm 0.07$ \\
\hline 30 m speed sprit time (s) & $4.23 \pm 0.09$ & $4.28 \pm 0.07$ & $4.20 \pm 0.09$ & $4.19 \pm 0.12$ \\
\hline $22 \mathrm{~m}$ agility sprint time (s) & $5.44 \pm 0.11$ & $5.51 \pm 0.17$ & $5.43 \pm 0.05$ & $5.37 \pm 0.07$ \\
\hline Counter movement jump height $(\mathrm{cm})$ & $40.0 \pm 5.3$ & $36.5 \pm 2.9$ & $41.0 \pm 5.5$ & $43.0 \pm 6.2$ \\
\hline Interval shuttle run test (shuttles) & $114 \pm 11$ & $110 \pm 14$ & $118 \pm 10$ & $112 \pm 8$ \\
\hline
\end{tabular}

Values presented are mean \pm standard deviation.

*Difference as follows: defenders $>$ (midfielders = attackers $)(p<0.01, E S>0.6)$. 


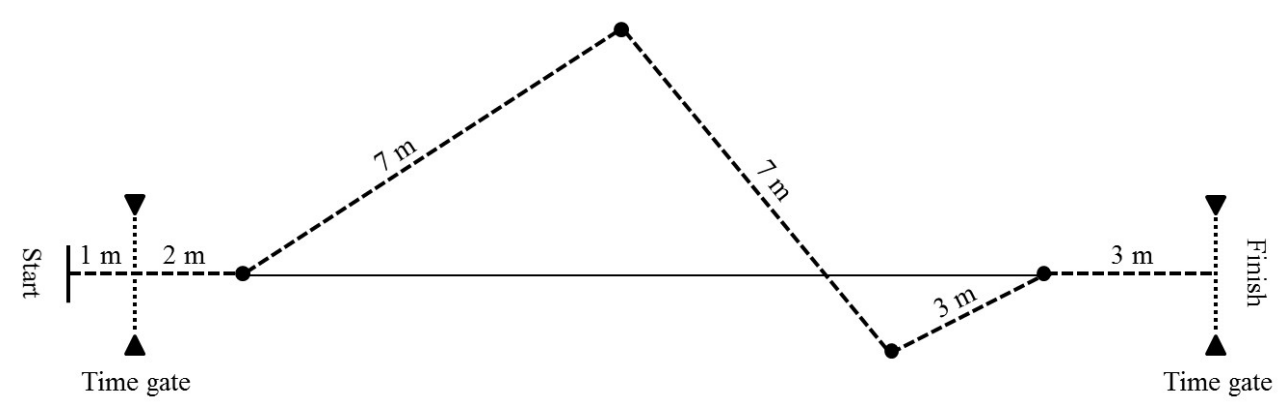

Figure 1

Design of the agility test.
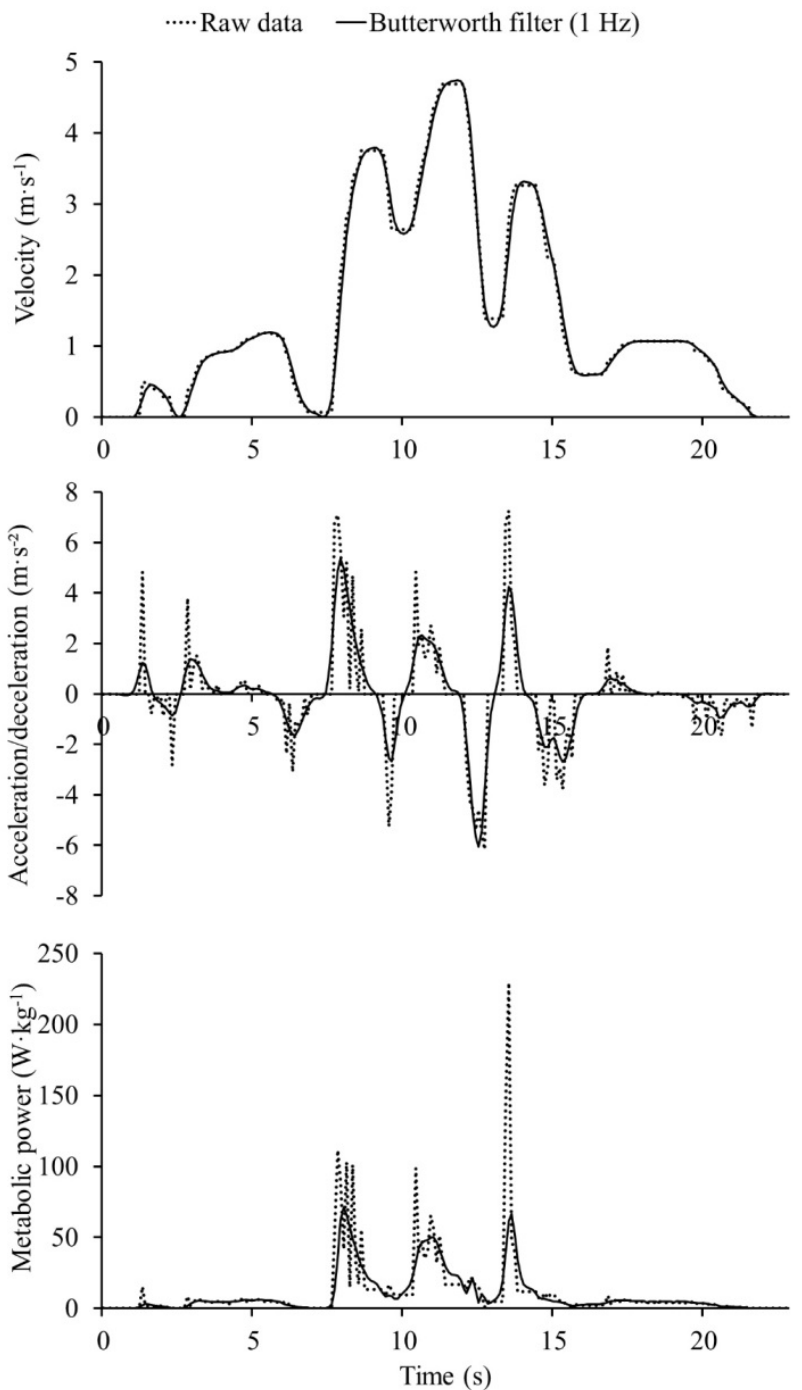

Figure 2

Impact of our applied data filtering technique on GPS-derived raw velocity, acceleration/deceleration and metabolic power data. The data are from one elite soccer player examined in the present study. 

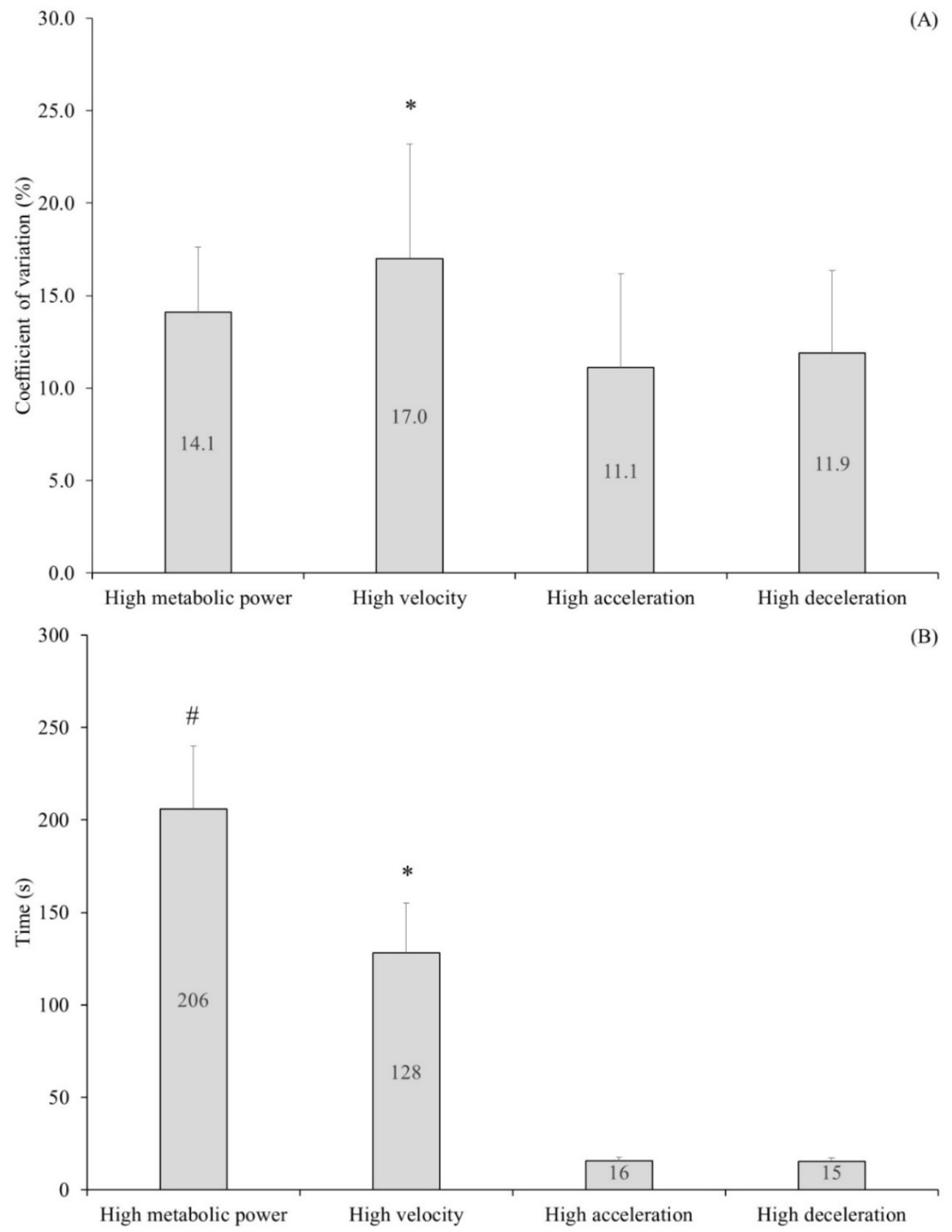

Figure 3

Within-subject variability of high metabolic power $\left(\geq 20 \mathrm{~W} \cdot \mathrm{kg}^{-1}\right)$, velocity $(\geq 15.5$ $\left.\mathrm{km} \cdot \mathrm{h}^{-1}\right)$, acceleration $\left(\geq 3 \mathrm{~m} \cdot \mathrm{s}^{-2}\right)$ and deceleration running $\left(\leq-3 \mathrm{~m} \cdot \mathrm{s}^{-2}\right)$ in elite soccer players $(n=12)$ during five pre-season matches $(A)$. Time spent above the corresponding thresholds is also shown (B).

Values presented are mean \pm standard deviation.

*Difference as follows: high velocity $>$ (high acceleration $=$ high deceleration) ( $p$ $<0.01, E S=0.3$ )

\#Differences as follows: high metabolic power $>$ high velocity $>$ (high acceleration $=$ high deceleration $)(p<0.01, E S=0.4)$. 

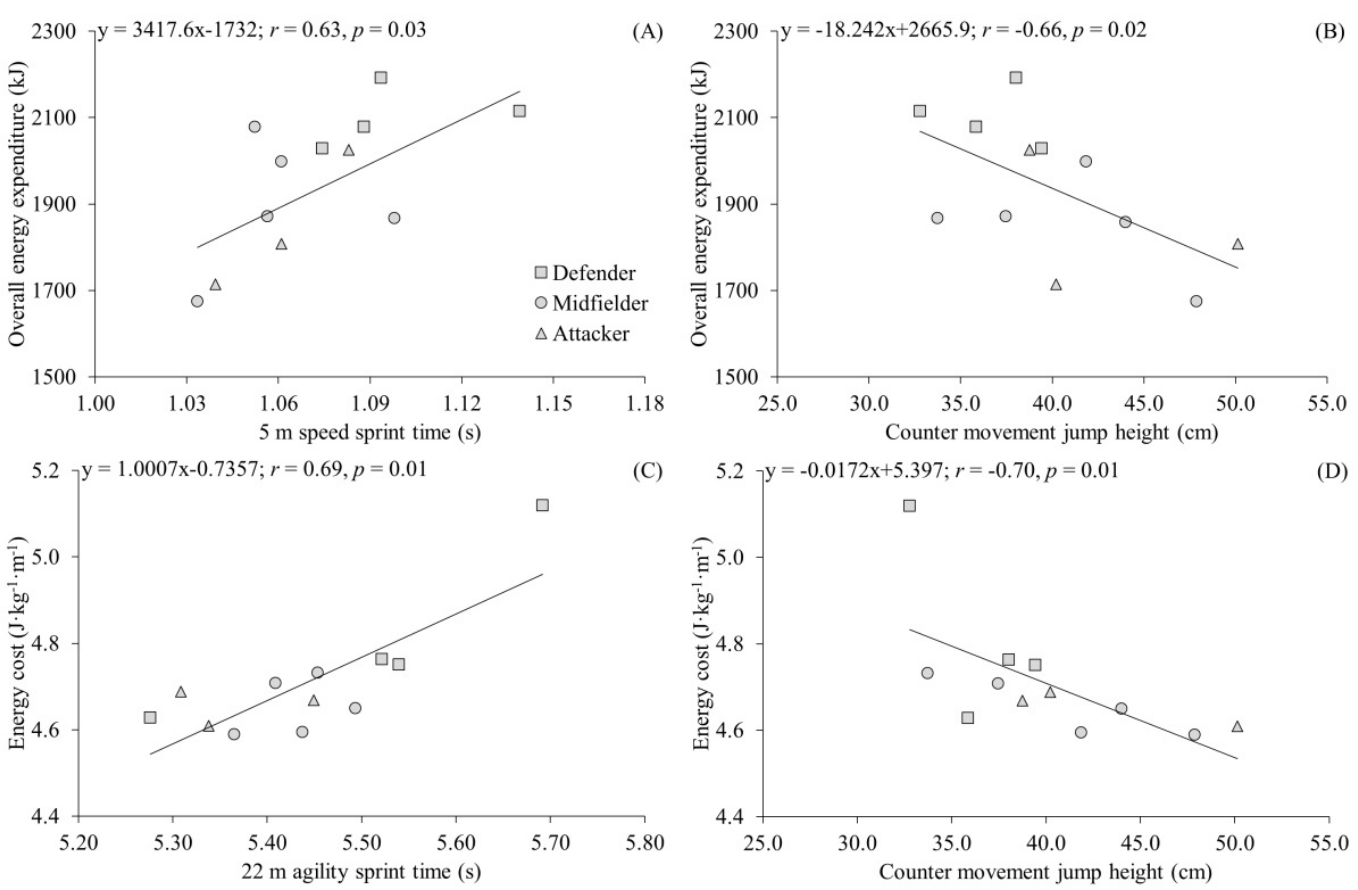

Figure 4

Relationships between metabolic power data from five pre-season matches and physical abilities in elite soccer players $(n=12)$.

The first novelty was that variability of global GPS-derived Pmet data such as the energy expenditure and cost and mean $P_{\text {met }}$ was lower than that for high-intensity including time spent $\geq 20 \mathrm{~W} \cdot \mathrm{kg}^{-1}$ and peak Pmet (Table 1). Our findings are supported by previous studies, which have revealed similar values and trends concerning the variability of camera-based velocity data in English Premier League and Italian Serie A players during official matches (Gregson et al., 2010; Rampinini et al., 2007). One explanation for these observations may be that high-intensity running is influenced more by situational (e.g., tactics, opponent, environmental conditions), internal (e.g., mechanical and metabolic loads, physical abilities, motivation) and technical sources (e.g., reliability of the technology), when compared to global running (Buchheit et al., 2014b; Gregson et al., 2010). Thus, variability of GPS-derived $P_{m e t}$ data in elite soccer players during pre-season matches is comparable to that previously reported for camera-based velocity data during official matches and increases with Pmet.

A further outcome was that variability of high $P_{\text {met }}$ was comparable to that of high velocity, acceleration and deceleration running, whereas variability of high velocity was moderately higher than that of high acceleration and deceleration running (Figure 3A). From a technical perspective, these findings were unexpected, because two previous studies had questioned the reliability of the GPS-technology for measuring acceleration and deceleration data (Buchheit et al., 2014a, 2014b), which, along with velocity data, are used to derive Pmet data (di Prampero et al., 2015). One reason for our findings may be that we eliminated the noise from the raw data through a filter before further calculations were computed (Figure 2), while such impacting data processing procedures (Akenhead et al., 2014) were not performed in previous 
studies (Buchheit et al., 2014a, 2014b). In consideration of the situational and internal perspective, our finding that variability of high velocity was moderately higher than that of high acceleration and deceleration running (Figure 3B) is plausible, because the latter two are fundamental demands to participate in soccer (Osgnach et al., 2010), whereas high velocity running may fluctuate with respect to the aforementioned situational (e.g., tactics) and internal factors (e.g., mechanical and metabolic loads) (Gregson et al., 2010).

Another novel finding was that defenders had a largely higher overall energy expenditure than midfielders and attackers. Defenders also had a largely greater body height, mass and fatfree mass (Table 2). Therefore, and in absence of further differences in $P_{\text {met }}$ data and total distance covered among playing positions (Table 2), the largely higher overall energy expenditure of the defenders is most likely a body mass effect and also reinforces the finding that the effort assessed only from velocity analyses is underestimated (Gaudino et al., 2014a, 2014b). These assumptions may have not only practical consequences with respect to the absolute energy intake of the defenders, but also to their medical/physical therapy following pre-season matches, because higher body mass as well as more frequent acceleration/deceleration induce higher musculoskeletal loads during play likewise (Silder et al., 2015). Since it has recently been reported that the GPS-derived Pmet approach underestimates the energy expenditure of soccerspecific drills compared to portable oxygen uptake measures (Buchheit et al., 2015), although these differences are negligible when the lag time for the aerobic metabolism is accounted for (Osgnach et al., 2016), more studies to verify our detected differences in energy expenditure are needed. However, it should be pointed out for the readers that instantaneous $P_{\text {met }}$ reflects the energy demand of only running based activities, while oxygen uptake indicates the aerobic energy supply and is also affected by other soccer activities such as jumps, kicks or tackles. Consequently, energy expenditure calculated from both approaches plausibly differs; hence, their combined application may provide a promising new framework to investigate instantaneous alterations in energy demand and supply in soccer (di Prampero et al., 2015; Osgnach et al., 2016).

Our finding that there were no further differences among playing positions (Table 2) contrasts with previous studies, showing that differences exist in both velocity match data (Bradley et al., 2009; Di Salvo et al., 2007) and physical abilities (Boone et al., 2012; Haugen et al., 2013). One explanation for these discrepancies is related to statistical calculations. In this study, there was a tendency for midfielders to cover more total distance and complete more shuttles, for attackers to reach higher peak Pmet, sprint faster and jump higher and for defenders to spend less time at high velocity running (Table 2). All these trends may have reached statistical significance with a larger sample size (Hopkins et al., 2009) and would have then supported previous studies (Boone et al., 2012; Bradley et al., 2009; Di Salvo et al., 2007; Haugen et al., 2013). However, from an applied soccer perspective, a further option is that, in elite players, and possibly with exception of central defenders (Di Salvo et al., 2013), there may currently exist more similarities than practical relevant differences in both match running demands and physical abilities among playing positions. Since technical/tactical factors have evolved more than physical ones during the last decade (Barnes et al., 2014), differentiate better between competitive standards (Bradley et al., 2013) and are associated with team success (Rampinini et al., 2009), it can be expected that the level and adaptiveness of the team tactics are presently main performance limiting factors (Hoppe et al., 2015b). Consequently, elite player's roles are less defined, and according to the needs of the team tactics, the players frequently change their positions not only across, but also during matches, particularly during pre-season matches as investigated here, and they also tend to attack/defend in collectives, which may explain our findings.

Our last novelty was that overall energy expenditure and cost were largely to very largely correlated with explosive physical abilities such as $5 \mathrm{~m}$ speed and $22 \mathrm{~m}$ agility sprint time and counter movement jump height (Figure 4). Our findings are supported by previous studies, showing, in male athletes during treadmill running, that energy costs were largely correlated with power of jumping (Lazzer et al., 2014) and 
that better economy was associated with higher strength of the plantar flexors (Arampatzis et al., 2006). Taken together, these findings suggest that explosive physical abilities of the lower extremities may favor the energy cost of both continuous and intermittent running, potentially due to anatomical (e.g., calcaneal tuberosity length), physiological (e.g., muscle fiber types), and biomechanical characteristics (e.g., energy storage and reuse of the muscle/connective tissue) (Lacour and Bourdin, 2015). Nevertheless, it is important to remember that energy expenditure and cost were computed here via a mathematical model from "chaotic" accelerated/decelerated running during soccer matches (Osgnach et al., 2010). Potentially, this could mean that more efficient players had lower overall activity levels during play, likely due to their superior explosive physical abilities, requiring longer periods to recover after intensive actions (Wallace and Norton, 2014). More research to elucidate these assumptions is necessary.

Although global $P_{\text {met }}$ data were moderately to largely correlated with explosive physical abilities, no relationships for highintensity $\mathrm{P}_{\mathrm{met}}$ data were detected. Conversely, in a previous study, it was shown that maximum oxygen uptake was moderately correlated with time spent $\geq 20 \mathrm{~W} \cdot \mathrm{kg}^{-1}$ in Italian Serie A players (Manzi et al., 2014). Interestingly, the magnitude of all correlations decreased from lower to higher $P_{m e t}$ categories, which was explained by a greater contribution from anaerobic pathways at higher Pmet (Manzi et al., 2014). Therefore, in our study, relationships with high-intensity $\mathrm{P}_{\mathrm{met}}$ data were expected considering that the assessed physical abilities were predominantly anaerobic (i.e, speed, agility and power) (Haugen et al., 2013) and aerobic-anaerobic (i.e., intermittent endurance) (Lemmink and Visscher, 2006) in nature. One possible reason for this discrepancy between our expected and observed results may be due to methodological limitations. Firstly, the Pmet algorithm neglects the air resistance (Osgnach et al., 2010), important at higher velocities (di Prampero et al., 2005), and allows only valid calculations for acceleration/deceleration data (i.e., $\geq-4.4$ to $\leq 4.4 \mathrm{~m} \cdot \mathrm{s}^{-2}$ ) corresponding to "equivalent slopes" (di Prampero et al., 2015; Osgnach et al., 2010) that lie within the range of treadmill inclinations investigated by Minetti et al. (2002). The second consideration is that the accuracy and reliability of our GPS-devices for measuring acceleration/deceleration data $\geq 4.0$ and $\leq-4.0 \mathrm{~m} \cdot \mathrm{s}^{-2}$ were limited (Akenhead et al., 2014) and that we applied a filtering technique to eliminate noise from the raw data (Figure 2) (Hoppe et al., 2014), potentially leading to a loss of information. All these methodological aspects may introduce biases with respect to $P_{\text {met }}$ data, which should be examined in future studies and may also explain our relatively low energy cost and high peak $\mathrm{P}_{\mathrm{met}}$ (Table 2) compared to the values reported for various sports in the literature (di Prampero et al., 2015). A further aspect from an applied soccer standpoint is that relationships between match running data and physical abilities are generally tactics and playing position driven, and therefore, complex to investigate (MendezVillanueva and Buchheit, 2011). Playing position specific relationships were not examined due to our small sample size, and more importantly, because no differences, with exception of overall energy expenditure and anthropometric measures, were found in both match running data and physical abilities among playing positions (Table 2), most likely due to the homogeneity of our sample. Finally, in contrast to endurance or strength athletes, elite soccer players do not need maximal, but rather optimally developed physical abilities to fulfill the demands of the team tactics (Hoppe et al., 2015b). Accordingly, linear relationships should not be expected in the present context.

While this study provides new knowledge, it is worth mentioning that our findings were limited by the circumstance that no competitive matches over $90 \mathrm{~min}$ were investigated. The particular reasons to investigate pre-season matches and examine only $45 \mathrm{~min}$ halves were that GPS-devices during Bundesliga or UEFA Europa League matches were not permitted, and secondly, coaches performed frequent substitutions to fine-tune the team tactics. However, it should be considered that our data were obtained from a unique population of which less empirical evidence is available, i.e., a sample of "true" elite soccer players including eight national players.

\section{Conclusions}

In conclusion, this study showed that, in 
elite soccer players across five pre-season matches, variability of global GPS-derived $P_{\text {met }}$ data such as energy expenditure was lower than that for high-intensity including time spent $\geq 20$ $\mathrm{W} \cdot \mathrm{kg}^{-1}$. However, variability of high Pmet running was comparable to that of high velocity, acceleration and deceleration running. Additionally, defenders had a largely higher overall energy expenditure than midfielders and attackers and overall energy expenditure and cost were largely to very largely correlated with explosive physical abilities of lower extremities. Therefore, it is concluded that GPS-derived Pmet analyses can provide new insights into the mechanics and energetics of soccer. Further research to improve the $P_{\text {met }}$ algorithm (di Prampero et al., 2015; Osgnach et al., 2010) and validate the GPS-technology, including filtering techniques (Akenhead et al., 2014; Hoppe et al., 2014), for measuring acceleration/deceleration (Buchheit et al., 2014a, 2014b) and Pmet (Buchheit et al., 2015; Rampinini et al., 2015) data is warranted. From a practical point of view, the detected variability suggests that global GPSderived $P_{\text {met }}$ data in elite soccer players from a single pre-season match should be preferably used for practical applications. Contrary, due to the observed large and uncertain variability in all high-intensity indicators, practitioners should interpret these data from single match observations cautiously and repeated measures are strongly recommended to establish meaningful high-intensity profiles of the players. Our findings also suggest that the absolute energy intake of defenders should be higher than that of other players to optimize recovery and adaptation processes following pre-season matches. Additionally, training drills and testing procedures for lower extremities incorporating energy storage and reuse mechanisms of the muscle/connective tissue, potentially favoring both players' explosive physical abilities and running economy during play, should be incorporated at an elite soccer level.

\section{Acknowledgements}

The authors are grateful to Joana Brochhagen for her helpful linguistic comments.

\section{References}

Akenhead R, French D, Thompson KG, Hayes PR. The acceleration dependent validity and reliability of 10 Hz GPS. J Sci Med Sport, 2014; 17: 562-566

Arampatzis A, De Monte G, Karamanidis K, Morey-Klapsing G, Stafilidis S, Bruggemann GP. Influence of the muscle-tendon unit's mechanical and morphological properties on running economy. J Exp Biol, 2006; 209: 3345-3357

Barnes C, Archer DT, Hogg B, Bush M, Bradley PS. The evolution of physical and technical performance parameters in the English Premier League. Int J Sports Med, 2014; 35: 1095-1100

Boone J, Vaeyens R, Steyaert A, Vanden Bossche L, Bourgois J. Physical fitness of elite Belgian soccer players by player position. J Strength Cond Res, 2012; 26: 2051-2057

Bradley PS, Carling C, Gomez Diaz A, Hood P, Barnes C, Ade J, Boddy M, Krustrup P, Mohr M. Match performance and physical capacity of players in the top three competitive standards of English professional soccer. Hum Mov Sci, 2013; 32: 808-821

Bradley PS, Sheldon W, Wooster B, Olsen P, Boanas P, Krustrup P. High-intensity running in English FA Premier League soccer matches. J Sports Sci, 2009; 27: 159-168

Buchheit M, Al Haddad H, Simpson BM, Palazzi D, Bourdon PC, Di Salvo V, Mendez-Villanueva A. Monitoring accelerations with GPS in football: time to slow down? Int J Sports Physiol Perform, 2014a; 9: 442-445 
Buchheit M, Allen A, Poon TK, Modonutti M, Gregson W, Di Salvo V. Integrating different tracking systems in football: multiple camera semi-automatic system, local position measurement and GPS technologies. J Sports Sci, 2014b; 32: 1844-1857

Buchheit M, Manouvrier C, Cassirame J, Morin JB. Monitoring Locomotor Load in Soccer: Is Metabolic Power, Powerful? Int J Sports Med, 2015; 36: 1149-1155

Carling C, Bloomfield J, Nelsen L, Reilly T. The role of motion analysis in elite soccer: contemporary performance measurement techniques and work rate data. Sports Med, 2008; 38: 839-862

Castellano J, Casamichana D, Calleja-Gonzalez J, Roman JS, Ostojic SM. Reliability and accuracy of $10 \mathrm{~Hz}$ GPS devices for short-distance exercise. J Sports Sci Med, 2011; 10: 233-234

di Prampero PE, Botter A, Osgnach C. The energy cost of sprint running and the role of metabolic power in setting top performances. Eur J Appl Physiol, 2015; 115: 451-469

di Prampero PE, Fusi S, Sepulcri L, Morin JB, Belli A, Antonutto G. Sprint running: a new energetic approach. J Exp Biol, 2005; 208: 2809-2816

Di Salvo V, Baron R, Tschan H, Calderon Montero FJ, Bachl N, Pigozzi F. Performance characteristics according to playing position in elite soccer. Int J Sports Med, 2007; 28: 222-227

Di Salvo V, Pigozzi F, Gonzalez-Haro C, Laughlin MS, De Witt JK. Match performance comparison in top English soccer leagues. Int J Sports Med, 2013; 34: 526-532

Dwyer DB, Gabbett TJ. Global positioning system data analysis: velocity ranges and a new definition of sprinting for field sport athletes. J Strength Cond Res, 2012; 26: 818-824

Gaudino P, Alberti G, Iaia FM. Estimated metabolic and mechanical demands during different small-sided games in elite soccer players. Hum Mov Sci, 2014a; 36: 123-133

Gaudino P, Iaia FM, Alberti G, Hawkins RD, Strudwick AJ, Gregson W. Systematic bias between running speed and metabolic power data in elite soccer players: influence of drill type. Int J Sports Med, 2014b; 35: 489-493

Gregson W, Drust B, Atkinson G, Salvo VD. Match-to-match variability of high-speed activities in premier league soccer. Int J Sports Med, 2010; 31: 237-242

Haugen TA, Tonnessen E, Seiler S. Anaerobic performance testing of professional soccer players 1995-2010. Int J Sports Physiol Perform, 2013; 8: 148-156

Hopkins WG. Measures of reliability in sports medicine and science. Sports Med, 2000; 30: 1-15

Hopkins WG, Marshall SW, Batterham AM, Hanin J. Progressive statistics for studies in sports medicine and exercise science. Med Sci Sports Exerc, 2009; 41: 3-13

Hoppe MW, Baumgart C, Bornefeld J, Sperlich B, Freiwald J, Holmberg HC. Running activity profile of adolescent tennis players during match play. Pediatr Exerc Sci, 2014; 26: 281-290

Hoppe MW, Baumgart C, Sperlich B, Ibrahim H, Jansen C, Willis SJ, Freiwald J. Comparison between three different endurance tests in professional soccer players. J Strength Cond Res, 2013; 27: 31-37

Hoppe MW, Freiwald J, Baumgart C, Born DP, Reed JL, Sperlich B. Relationship between core strength and key variables of performance in elite rink hockey players. J Sports Med Phys Fitness, 2015a; 55: 150-157

Hoppe MW, Slomka M, Baumgart C, Weber H, Freiwald J. Match running performance and success across a season in German Bundesliga soccer teams. Int J Sports Med, 2015b; 36: 563-566

Lacour JR, Bourdin M. Factors affecting the energy cost of level running at submaximal speed. Eur J Appl Physiol, 2015; 115: 651-673

Lazzer S, Taboga P, Salvadego D, Rejc E, Simunic B, Narici MV, Buglione A, Giovanelli N, Antonutto G, 
Grassi B, Pisot R, di Prampero PE. Factors affecting metabolic cost of transport during a multi-stage running race. J Exp Biol, 2014; 217: 787-795

Lemmink KA, Visscher C, Lambert MI, Lamberts RP. The interval shuttle run test for intermittent sport players: evaluation of reliability. J Strength Cond Res, 2004; 18: 821-827

Lemmink KA, Visscher SH. Role of energy systems in two intermittent field tests in women field hockey players. J Strength Cond Res, 2006; 20: 682-688

Manzi V, Impellizzeri F, Castagna C. Aerobic fitness ecological validity in elite soccer players: a metabolic power approach. J Strength Cond Res, 2014; 28: 914-919

Mendez-Villanueva A, Buchheit M. Physical capacity-match physical performance relationships in soccer: simply, more complex. Eur J Appl Physiol, 2011; 111: 2387-2389

Minetti AE, Moia C, Roi GS, Susta D, Ferretti G. Energy cost of walking and running at extreme uphill and downhill slopes. J Appl Physiol (1985), 2002; 93: 1039-1046

Osgnach C, Paolini E, Roberti V, Vettor M, di Prampero PE. Metabolic power and oxygen consumption in team sports: A brief response to Buchheit et al. Int J Sports Med, 2016; 37: 77-81

Osgnach C, Poser S, Bernardini R, Rinaldo R, di Prampero PE. Energy cost and metabolic power in elite soccer: a new match analysis approach. Med Sci Sports Exerc, 2010; 42: 170-178

Pallant J. SPSS survival manual - a step by step guide to data analysis using SPSS. Maidenhead: McGraw-Hill 2010.

Polglaze T, Dawson B, Peeling P. Gold standard or fool's gold? The efficacy of displacement variables as indicators of energy expenditure in team sports. Sports Med, 2016; 46: 657-670

Rampinini E, Alberti G, Fiorenza M, Riggio M, Sassi R, Borges TO, Coutts AJ. Accuracy of GPS devices for measuring high-intensity running in field-based team sports. Int J Sports Med, 2015; 36: 49-53

Rampinini E, Coutts AJ, Castagna C, Sassi R, Impellizzeri FM. Variation in top level soccer match performance. Int J Sports Med, 2007; 28: 1018-1024

Rampinini E, Impellizzeri FM, Castagna C, Coutts AJ, Wisloff U. Technical performance during soccer matches of the Italian Serie A league: effect of fatigue and competitive level. J Sci Med Sport, 2009; 12: $227-233$

Silder A, Besier T, Delp SL. Running with a load increases leg stiffness. J Biomech, 2015; 48: 1003-1008

Varley MC, Fairweather IH, Aughey RJ. Validity and reliability of GPS for measuring instantaneous velocity during acceleration, deceleration, and constant motion. J Sports Sci, 2012; 30: 121-127

Wallace JL, Norton KI. Evolution of World Cup soccer final games 1966-2010: game structure, speed and play patterns. J Sci Med Sport, 2014; 17: 223-228

\section{Corresponding author:}

\section{Matthias W. Hoppe}

University of Wuppertal, Department of Movement and Training Science

Fuhlrottstaße 10, 42119 Wuppertal, Germany

Phone: +49 (0)202 4393128

E-mail: m.hoppe@uni-wuppertal.de 